\title{
NON-EXISTENCE AND UNIQUENESS OF LIMIT CYCLES FOR PLANAR POLYNOMIAL DIFFERENTIAL SYSTEMS WITH HOMOGENEOUS NONLINEARITIES
}

\author{
JIANFENG HUANG ${ }^{1}$, HAIHUA LIANG ${ }^{2}$ AND JAUME LLIBRE ${ }^{3}$
}

\begin{abstract}
In this paper we study the limit cycles of the planar polynomial differential systems

$$
\begin{aligned}
& \dot{x}=a x-y+P_{n}(x, y), \\
& \dot{y}=x+a y+Q_{n}(x, y),
\end{aligned}
$$

where $P_{n}$ and $Q_{n}$ are homogeneous polynomials of degree $n \geq 2$, and $a \in \mathbb{R}$. Consider the functions

$$
\begin{aligned}
& \varphi(\theta)=P_{n}(\cos \theta, \sin \theta) \cos \theta+Q_{n}(\cos \theta, \sin \theta) \sin \theta \\
& \psi(\theta)=Q_{n}(\cos \theta, \sin \theta) \cos \theta-P_{n}(\cos \theta, \sin \theta) \sin \theta, \\
& \omega_{1}(\theta)=a \psi(\theta)-\varphi(\theta), \\
& \omega_{2}(\theta)=(n-1)(2 a \psi(\theta)-\varphi(\theta))+\psi^{\prime}(\theta) .
\end{aligned}
$$

First we prove that these differential systems have at most 1 limit cycle if there exists a linear combination of $\omega_{1}$ and $\omega_{2}$ with definite sign. This result improves previous knwon results. Furthermore, if $\omega_{1}\left(\nu_{1} a \psi-\nu_{2} \varphi\right) \leq 0$ for some $\nu_{1}, \nu_{2} \geq 0$, we provide necessary and sufficient conditions for the non-existence, and the existence and uniqueness of the limit cycles of these differential systems. When one of these mentioned limit cycles exists it is hyperbolic and surrounds the origin.
\end{abstract}

\section{INTRODUCTION AND STATEMENTS OF MAIN RESULTS}

In this work we study the limit cycles of the planar polynomial differential systems with homogeneous nonlinearities of the form

$$
\begin{aligned}
& \frac{d x}{d t}=\dot{x}=a x-y+P_{n}(x, y), \\
& \frac{d y}{d t}=\dot{y}=x+a y+Q_{n}(x, y),
\end{aligned}
$$

where $P_{n}$ and $Q_{n}$ are homogeneous polynomials of degree $n \geq 2$. The study of the limit cycles of these differential systems is a particular case of the second part of Hilbert's 16 th problem, which is one of the main problems in the qualitative theory of the planar polynomial differential systems.

2010 Mathematics Subject Classification. Primary 34C07. Secondary 37C05. Tertiary 35F60.

Key words and phrases. Limit cycles; non-existence and uniqueness; polynomial differential systems; homogeneous nonlinearities. 
These last decades the differential systems (1) have been extensively studied and gained wide attention. This is mainly due to the fact that each limit cycle surrounding the origin of a system (1) can be expressed in polar coordinates as $r=r(\theta)$, with $r(\theta)$ being a smooth periodic function. More precisely, system (1) in the polar coordinates $(r, \theta)$ given by $x=r \cos \theta, y=r \sin \theta$ can be written in the form

$$
\begin{aligned}
& \dot{r}=a r+\varphi(\theta) r^{n}, \\
& \dot{\theta}=1+\psi(\theta) r^{n-1} .
\end{aligned}
$$

And it is known that the limit cycles surrounding the origin of system (1) do not intersect the curve $\dot{\theta}=1+\psi(\theta) r^{n-1}=0$ (see [11, 16, 23, 24], etc). Therefore these limit cycles can be investigated using the differential equation

$$
\frac{d r}{d \theta}=\frac{a r+\varphi(\theta) r^{n}}{1+\psi(\theta) r^{n-1}}, \quad \theta \in[0,2 \pi] .
$$

Such particularity provides an opportunity to analyze the Hilbert's 16th problem restricted to the limit cycles surrounding the origin of the differential systems (1) in a relatively simple way.

There are several works studying the limit cycles which bifurcate from the ones of the differential systems (1) under small perturbations, see for instance $[5,24,33,34,38]$ and the references therein. But here we are interested in the results for the non-bifurcation case, now we summarize the more representatives.

(I) If $\omega_{1}(\theta) \not \equiv 0$ does not change sign, then the differential system (1) has at most 1 limit cycle in the region $\dot{\theta}>0$ surrounding the origin (see Coll, Gasull and Prohens [16]).

(II) If $\omega_{1}(\theta) \psi(\theta) \not \equiv 0$ does not change sign, then the differential system (1) has at most 1 (resp. 2) limit cycle(s) in the region $\dot{\theta}>0$ surrounding the origin when $n$ is even (resp. odd) (see Carbonell and Llibre [11]).

(III) If $\omega_{2}(\theta) \not \equiv 0$ does not change sign, then the differential system (1) has at most 2 limit cycles in the region $\dot{\theta}>0$ surrounding the origin (see Gasull and Llibre [23]).

(IV) If either $\omega_{1}(\theta) \psi(\theta) \equiv 0$, or $\omega_{2}(\theta) \equiv 0$, then the differential system (1) has at most 1 limit cycle in the region $\dot{\theta}>0$ surrounding the origin (see Gasull and Llibre [23]).

For more works on the differential system (1), see [13, 14, 19, 32, 35, 36], etc.

The main goal of this paper is to provide some new criteria for the uniqueness and non-existence of the limit cycles of the differential system (1).

Theorem 1. Suppose there exist $\mu_{1}, \mu_{2} \in \mathbb{R}$ such that $\mu_{1}^{2}+\mu_{2}^{2} \neq 0$ and $\mu_{1} \omega_{1}(\theta)+$ $\mu_{2} \omega_{2}(\theta) \geq 0(\leq 0)$.

(a) If $\psi(\theta)>0$ and $a \int_{0}^{2 \pi} \frac{\varphi(\theta)}{\psi(\theta)} d \theta \geq 0$, then system (1) has no limit cycles. 
(b) If $\psi(\theta)>0$ and $a \int_{0}^{2 \pi} \frac{\varphi(\theta)}{\psi(\theta)} d \theta<0$, then system (1) has exactly one limit cycle.

(c) If either $\psi(\theta)<0$ or $\psi(\theta)$ has zeros, then system (1) has at most one limit cycle. This upper bound is sharp.

Moreover when such a limit cycle exists, it is hyperbolic and surrounds the origin.

We remark that Theorem 1 generalizes the known results (I) and (III), and provides more information about the result (III) under additional assumptions. Furthermore we will show in Proposition 13 that, the condition $\mu_{1} \omega_{1}+\mu_{2} \omega_{2} \geq 0(\leq 0)$ in Theorem 1 , actually leads to the "tangency" or "transversality" of the vector field defined by the differential system (1) on an algebraic curve. This provides a geometric meaning to this condition. Conversely, if this "tangency" or "transversality" is valid, then in some case we can obtain the best upper bound for the number of limit cycles of the system, see the following corollary.

Corollary 2. Suppose $\psi(\theta) \neq 0$ and there exists $\mu \in \mathbb{R} \backslash\{0\}$ such that $\psi(\theta) r^{n-1}+\mu=0$ is an invariant curve or a transversal section for the vector field of system (2). Then the differential system (1) has at most one limit cycle. Moreover when this limit cycle exists it is hyperbolic and surrounds the origin.

We remark that Corollary 2 says that the "tangency" or "transversality" of the vector field of system (1) on the algebraic curves $\left(Q_{n} x-P_{n} y\right)+\mu\left(x^{2}+y^{2}\right)=0$ with $\mu \in \mathbb{R} \backslash\{0\}$ and $(x, y) \neq(0,0)$, provides useful information to know the uniqueness of the limit cycles of the differential system (1). This result is quite similar to the classical result, which says that a quadratic polynomial differential system has at most 1 limit cycle if it has an invariant straight line, see $[17,18,40]$.

We also have a partial improvement for the known result (II), see the next theorem.

Theorem 3. Suppose there exist $\nu_{1}, \nu_{2} \geq 0$ such that $\left(a \nu_{1}\right)^{2}+\nu_{2}^{2} \neq 0$ and $\omega_{1}\left(\nu_{1} a \psi(\theta)-\right.$ $\left.\nu_{2} \varphi(\theta)\right) \leq 0$. Then the following statements hold.

(i) If $\omega_{1} \varphi \equiv 0$, then system (1) has no limit cycles.

(ii) If $\omega_{1} \varphi \not \equiv 0$ and $\psi(\theta)>0$, then system (1) has no limit cycles.

(iii) If $\omega_{1} \varphi \not \equiv 0$ and $\psi(\theta)<0$, then system (1) has at most one limit cycle. In addition, such a limit cycle exactly exists if $\omega_{1} \neq 0$.

(iv) If $\omega_{1} \varphi \not \equiv 0, \psi(\theta)$ has zeros and $\nu_{1} \leq \nu_{2}$, then system (1) has no limit cycles.

(v) If $\omega_{1} \varphi \not \equiv 0, \psi(\theta)$ has zeros and $\nu_{1}>\nu_{2}$, then system (1) has at most one limit cycle.

Moreover when such a limit cycle exists, it is hyperbolic and surrounds the origin.

In what follows by choosing different values for the parameters $\mu_{1}, \mu_{2}, \nu_{1}$ and $\nu_{2}$ in Theorems 1 and 3, we recover some of the classical results on the limit cycles of the differential systems (1), and also some new ones. 
Corollary 4. The polynomial differential system (1) has at most one limit cycle if one of the following conditions holds. Moreover when this limit cycle exists, it is hyperbolic and surrounds the origin.

(i) $a \psi(\theta)-\varphi(\theta) \geq 0(\leq 0)$, i.e. $\omega_{1}(\theta) \geq 0(\leq 0)$.

(ii) $(n-1)(2 a \psi(\theta)-\varphi(\theta))+\psi^{\prime}(\theta) \geq 0(\leq 0)$, i.e. $\omega_{2}(\theta) \geq 0(\leq 0)$.

(iii) $(n-1) a \psi(\theta)+\psi^{\prime}(\theta) \geq 0(\leq 0)$, i.e. $-(n-1) \omega_{1}(\theta)+\omega_{2}(\theta) \geq 0(\leq 0)$.

(iv) $(n-1) \varphi(\theta)+\psi^{\prime}(\theta) \geq 0(\leq 0)$, i.e. $-2(n-1) \omega_{1}(\theta)+\omega_{2}(\theta) \geq 0(\leq 0)$.

(v) $(a \psi(\theta)-\varphi(\theta)) a \psi(\theta) \leq 0$ (i.e. $a \omega_{1}(\theta) \psi(\theta) \leq 0$ ) with $a \neq 0$.

(vi) $(a \psi(\theta)-\varphi(\theta)) \varphi(\theta) \geq 0$, i.e. $\omega_{1}(\theta) \varphi(\theta) \geq 0$.

(vii) $\nu_{1} a \psi(\theta)-\nu_{2} \varphi(\theta) \equiv 0$ with $\nu_{1}, \nu_{2} \geq 0$ and $a \nu_{1}^{2}+\nu_{2}^{2} \neq 0$.

Clearly statement (i) of Corollary 4 includes the result (I) and part of the result (IV). Statement (ii) of Corollary 4 is an improvement of the result (III). The result (IV) follows from statements (i), (ii) and (v). Finally statement (v) of Corollary 4 partially improves the result (II).

We provide three applications of our results. The first one shows the uniqueness of limit cycles and compares our results with the results (I)-(IV). The second one studies the interval of the parameter $a$, in which the differential system (1) has no no limit cycles, or exactly one limit cycle. The last application concerns with the non-existence of limit cycles. These applications are done in section 3.

There are two powerful tools in the proof of our main results. One of them is the Abel differential equation

$$
\frac{d x}{d t}=S(t, x)=A(t) x^{3}+B(t) x^{2}+C(t) x,
$$

where $A(t), B(t)$ and $C(t)$ are $C^{\infty}([0, \kappa])$ functions with $\kappa>0$, and $x \in \mathbb{R}$, because using the transformation introduced by Cherkas [12], which really goes back to Liouville [31],

$$
\rho=\frac{r^{n-1}}{1+\psi(\theta) r^{n-1}},
$$

the differential equation (3) becomes the Abel differential equation

$$
\frac{d \rho}{d \theta}=\mathcal{S}(\theta, \rho)=(n-1)(a \psi-\varphi) \psi \rho^{3}+((n-1)(\varphi-2 a \psi)-\dot{\psi}) \rho^{2}+(n-1) a \rho .
$$

There are many works on the Abel differential equation because of its importance in the qualitative theory of the differential equations, see $[1-4,6-10,15,19,21-23,27-$ $30,37,39]$ and the references therein. Nevertheless, up to now, the major part of results on the limit cycles of the Abel differential equations require that some coefficient of the Abel differential equation does not change sign. We want to point out that the proofs of our theorems use a result of [25] on the Abel differential equations where some coefficients can change their signs.

On the other hand we will see that the results on the limit cycles of the Abel differential equations not always can be used, especially when the function $\psi(\theta)$ has zeros. 
In such case we have to investigate directly the differential equation (3), which seems more difficult. Usually in order to control the maximum number of limit cycles for such equations, an efficient way is to find some suitable auxiliary functions, see for example Theorem 5 of [4], or Lemma 2.1 of [25], or Proposition 2.2 of [28].

The second main tool that we shall use in this paper, is a formula on the divergence from [26]. According to this formula, if the auxiliary function for the Abel differential equation (6) is known when $\psi(\theta) \neq 0$, then we can obtain an auxiliary function for the differential equation (3) when the function $\psi(\theta)$ has zeros. In this way the proofs using the differential equation (3) can be done in a similar way to the proofs using the Abel differential equation (6).

The organization of this paper is as follows. In section 2 we provide some preliminary results. The proofs of Theorems 1 and 3, and of Corollaries 2 and 4 are given in section 3.

\section{Preliminaries}

For proving our theorems and corollaries we need some preliminary results, we state them one by one in the following.

2.1. Some basic definitions and results. Consider the differential equation

$$
\frac{d x}{d t}=L(t, x),
$$

where $L \in \mathrm{C}^{\infty}([0, \kappa] \times \mathbb{R})$ and $\kappa$ is a positive constant. Denote by $x\left(t, x_{0}\right)$ the solution of (7) such that $x\left(0, x_{0}\right)=x_{0}$.

The solution $x\left(t, x_{0}\right)$ is a periodic solution of the differential equation (7), if it is defined in $[0, \kappa]$ with $x\left(\kappa, x_{0}\right)=x_{0}$. Moreover, an orbit $\left(t, x\left(t, x_{0}\right)\right)$ in the strip $[0, \kappa] \times \mathbb{R}$ is a periodic orbit if $x\left(t, x_{0}\right)$ is a periodic solution of equation (7). A periodic orbit is a limit cycle if it is isolated in the set of all periodic orbits of the differential equation (7).

The function $H\left(x_{0}\right)=x\left(\kappa, x_{0}\right)$ is the return map of the differential equation (7). It is well-known that

$$
H^{\prime}\left(x_{0}\right)=\exp \int_{0}^{\kappa} \frac{\partial L}{\partial x}\left(t, x\left(t, x_{0}\right)\right) d t
$$

where the prime represents the first-order derivative (see Lloyd [37] for instance). When the solution $x\left(t, x_{0}\right)$ is periodic with $H^{\prime}\left(x_{0}\right) \neq 1\left(\operatorname{resp} . \quad(H-i d)^{\prime}\left(x_{0}\right)=\cdots=(H-\right.$ $i d)^{(n-1)}\left(x_{0}\right)=0$ and $\left.(H-i d)^{(n)}\left(x_{0}\right) \neq 0\right)$, the solution $x\left(t, x_{0}\right)$ provides a limit cycle, which is called a hyperbolic limit cycle (resp. a limit cycle with multiplicity $n$ ). For more details see for instance [20].

Clearly a limit cycle of equation (7) is hyperbolic if and only if it has multiplicity 1.

Lemma 5. Let $U=\left\{(t, x): t \in[0, \kappa], x \in\left(c_{1}(t), c_{2}(t)\right)\right\}$ where $c_{i}:[0, \kappa] \rightarrow \mathbb{R} \cup$ $\{+\infty,-\infty\}$ for $i=1,2$ are two functions. Let $F \in \mathrm{C}^{1}(U)$ with $F(\kappa, x)=F(0, x)$. 
Consider the function $G: U \rightarrow \mathbb{R}$ defined by

$$
G(t, x)=\frac{\partial L}{\partial x}(t, x)+\frac{\partial F}{\partial t}(t, x)+L(t, x) \frac{\partial F}{\partial x}(t, x) .
$$

(i) If $x=x(t)$ is a periodic solution of the differential equation (7) in $U$, then

$$
\int_{0}^{\kappa} \frac{\partial L}{\partial x}(t, x(t)) d t=\int_{0}^{\kappa} G(t, x(t)) d t .
$$

(ii) If $\left.G\right|_{U} \geq 0$ (resp. $\leq 0$ ) and there exists a non-empty open set $E \subseteq[0, \kappa]$ such that $\left.G\right|_{U \cap(E \times \mathbb{R})} \neq 0$, then the differential equation (7) has at most 1 limit cycle in $U$, which is hyperbolic and unstable (resp. stable).

The proof of Lemma 5 is easy, and it follows in a similar way to the proof of Lemma 2.1 of [25].

Now we state a second result which follows essentially from Lemma 5 . We denote by $W(\cdot, \cdot)$ the Wronskian determinant of two functions.

Theorem 6. Suppose there exist two smooth functions $\lambda_{1}(t)>\lambda_{2}(t)$ such that for $i=1,2$ we have that $\lambda_{i}(t) \neq 0, \lambda_{i}(\kappa)=\lambda_{i}(0), S\left(t, \lambda_{i}(t)\right)-\dot{\lambda}_{i}(t)$ does not change signs, and

$$
4 \lambda_{1}(t) \lambda_{2}(t)\left(S\left(t, \lambda_{1}(t)\right)-\dot{\lambda_{1}}(t)\right)\left(S\left(t, \lambda_{2}(t)\right)-\dot{\lambda_{2}}(t)\right)+W^{2}\left(\lambda_{1}(t), \lambda_{2}(t)\right) \leq 0 .
$$

Then the Abel differential equation (4) has at most 2 non-zero limit cycles, counted with multiplicities.

Theorem 6 with $\kappa=1$ is Theorem 1.1 of [25]. And it can be obtained by rescaling $t \mapsto t / \kappa$ when $\kappa \neq 1$.

In any case we want to sketch the proof of Theorem 6 , which will be helpful for proving our Theorem 1. It is easy to verify that Theorem 6 holds if $S\left(t, \lambda_{1}(t)\right)-\dot{\lambda}_{1}(t)=$ $S\left(t, \lambda_{2}(t)\right)-\dot{\lambda_{2}}(t)=W\left(\lambda_{1}(t), \lambda_{2}(t)\right) \equiv 0$. When $S\left(t, \lambda_{1}(t)\right)-\dot{\lambda_{1}}(t), S\left(t, \lambda_{2}(t)\right)-\dot{\lambda_{2}}(t)$ and $W\left(\lambda_{1}(t), \lambda_{2}(t)\right)$ are not all identically zero, the key point is to find a suitable function $F(t, x)$ and then apply Lemma 5. Define $f(t, x)=\left(x-\lambda_{1}(t)\right)\left(x-\lambda_{2}(t)\right) x$. Based on the Lagrange interpolation formula, the authors in [25] take

$$
F(t, x)=-\ln \left|\frac{f(t, x)}{\lambda_{1}(t) \lambda_{2}(t)}\right|, \text { where }(t, x) \in\left\{(t, x): x \notin\left\{0, \lambda_{1}(t), \lambda_{2}(t)\right\}\right\} .
$$

Therefore a direct calculation shows that

$$
\frac{\partial S}{\partial x}(t, x)+\frac{\partial F}{\partial t}(t, x)+S(t, x) \frac{\partial F}{\partial x}(t, x)=f(t, x) \frac{I_{S}(t, x)}{\lambda_{1}-\lambda_{2}},
$$

where

$$
I_{S}(t, x)=\sum_{i=1}^{2} \frac{(-1)^{i}\left(S\left(t, \lambda_{i}(t)\right)-\dot{\lambda}_{i}(t)\right)}{\lambda_{i}(t)\left(x-\lambda_{i}(t)\right)^{2}}-\frac{W\left(\lambda_{1}(t), \lambda_{2}(t)\right)}{\lambda_{1}(t) \lambda_{2}(t)\left(x-\lambda_{1}(t)\right)\left(x-\lambda_{2}(t)\right)} .
$$

It is easy to prove that $\left.I_{S}\right|_{U} \geq 0(\leq 0)$, where $U$ is an arbitrary connected component of $\left\{(t, x): x \notin\left\{0, \lambda_{1}(t), \lambda_{2}(t)\right\}\right\}$. Moreover there exists a non-empty open set $E \subseteq[0, \kappa]$ 
such that $\left.I_{S}\right|_{U \cap(E \times \mathbb{R})} \neq 0$. Note that $\left.f\right|_{U} \neq 0$. By Lemma $5 U$ contains at most 1 limit cycle of the differential equation (7). Therefore the number of non-zero limit cycles of (7) is no more than 6 (2 in the curves $x=\lambda_{1}(t)$ and $x=\lambda_{2}(t)$, and 4 coming at most one for each connected components of the set $\left.\left\{(t, x): x \notin\left\{0, \lambda_{1}(t), \lambda_{2}(t)\right\}\right\}\right)$. By virtue of further analysis (including bifurcation method and comparison principle), this upper bound can be reduced to 2 and it is sharp. Thus we obtain Theorem 6 , for more details see [25].

There is no doubt that the Abel differential equation is a powerful tool for studying the limit cycles of the differential system (1). However not always we can use it. For this reason we also need to apply Lemma 5 directly to the differential equation (3), but the difficulty is still to find a suitable auxiliary function for applying the Lemma 5 to equation (3). To this end we introduce the following result given in [26].

Lemma 7. Let $V_{1}$ and $V_{2}$ be two open subsets of $\mathbb{R}^{2}$, let $T: V_{1} \rightarrow V_{2}$ be a diffeomorphism, and let $F: V_{2} \rightarrow \mathbb{R}$ be a $C^{1}$ function. Assume that $\mathbf{Q}$ and $\mathbf{P}$ are two vector fields on $V_{1}$ and $V_{2}$, respectively, with $\mathbf{P} \circ T=D T \mathbf{Q}$. Then

$$
\left(\operatorname{div} \mathbf{P}+D_{\mathbf{P}} F\right) \circ T=\operatorname{div} \mathbf{Q}+D_{\mathbf{Q}} \bar{F},
$$

where $D_{\mathbf{Q}}$ represents the directional derivative along $\mathbf{Q}$ and $\bar{F}=\ln |D T|+F \circ T$.

Proof. Denote by $\mathbf{Q}=\left(\mathrm{Q}_{1}, \mathrm{Q}_{2}\right), \mathbf{P}=\left(\mathrm{P}_{1}, \mathrm{P}_{2}\right)$ and $\boldsymbol{y}=\left(y_{1}, y_{2}\right)=T(\boldsymbol{x})=T\left(x_{1}, x_{2}\right)$. We have $\mathbf{P}(\boldsymbol{y})=(D T \cdot \mathbf{Q})(\boldsymbol{x})$. Hence a straightforward calculation shows that

$$
\begin{aligned}
& \mathrm{P}_{1 y_{1}} y_{1 x_{1}}+\mathrm{P}_{1 y_{2}} y_{2 x_{1}}=y_{1 x_{1} x_{1}} \mathrm{Q}_{1}+y_{1 x_{1} x_{2}} \mathrm{Q}_{2}+y_{1 x_{1}} \mathrm{Q}_{1 x_{1}}+y_{1 x_{2}} \mathrm{Q}_{2 x_{1}}, \\
& \mathrm{P}_{2 y_{1}} y_{1 x_{1}}+\mathrm{P}_{2 y_{2}} y_{2 x_{1}}=y_{2 x_{1} x_{1}} \mathrm{Q}_{1}+y_{2 x_{1} x_{2}} \mathrm{Q}_{2}+y_{2 x_{1}} \mathrm{Q}_{1 x_{1}}+y_{2 x_{2}} \mathrm{Q}_{2 x_{1}}, \\
& \mathrm{P}_{1 y_{1}} y_{1 x_{2}}+\mathrm{P}_{1 y_{2}} y_{2 x_{2}}=y_{1 x_{1} x_{2}} \mathrm{Q}_{1}+y_{1 x_{2} x_{2}} \mathrm{Q}_{2}+y_{1 x_{1}} \mathrm{Q}_{1 x_{2}}+y_{1 x_{2}} \mathrm{Q}_{2 x_{2}}, \\
& \mathrm{P}_{2 y_{1}} y_{1 x_{2}}+\mathrm{P}_{2 y_{2}} y_{2 x_{2}}=y_{2 x_{1} x_{2}} \mathrm{Q}_{1}+y_{2 x_{2} x_{2}} \mathrm{Q}_{2}+y_{2 x_{1}} \mathrm{Q}_{1 x_{2}}+y_{2 x_{2}} \mathrm{Q}_{2 x_{2}} .
\end{aligned}
$$

So, $y_{2 x_{2}} \times(12)-y_{1 x_{2}} \times(13)-y_{2 x_{1}} \times(14)+y_{1 x_{1}} \times(15)$ leads to

$$
|D T|\left(\mathrm{P}_{1 y_{1}}+\mathrm{P}_{2 y_{2}}\right)=|D T|_{x_{1}} \mathrm{Q}_{1}+|D T|_{x_{2}} \mathrm{Q}_{2}+|D T|\left(\mathrm{Q}_{1 x_{1}}+\mathrm{Q}_{2 x_{2}}\right),
$$

which implies

$$
(\operatorname{div} \mathbf{P}) \circ T=\operatorname{div} \mathbf{Q}+D_{\mathbf{Q}} \ln |D T| .
$$

Furthermore, observe that

$$
D_{\mathbf{P}} F \circ T=(\nabla F \circ T) \cdot(\mathbf{P} \circ T)=(\nabla F \circ T) \cdot D T \cdot \mathbf{Q}=\nabla(F \circ T) \cdot \mathbf{Q}=D_{\mathbf{Q}}(F \circ T) .
$$

The conclusion of the lemma follows.

For ending this subsection we state two more lemmas. The first provides information on the stability at infinity for the polynomial differential system (1) when $\psi(\theta) \neq 0$ (it is known that the infinity is a periodic orbit of the system in this case), the second provides the non-existence of limit cycles under convenient assumptions. 
Lemma 8. Let $\varphi(\theta)$ and $\psi(\theta)$ be the functions defined in the abstract. If $\psi(\theta) \neq 0$ and

$$
\int_{0}^{2 \pi} \frac{\varphi(\theta)}{|\psi(\theta)|} d \theta>0(\text { resp. }<0)
$$

then the periodic orbit at the infinity of the polynomial differential system (1) is stable (resp. unstable).

Lemma 8 is in fact a particular case of Proposition 4 of [24].

Lemma 9. Let $l \in \mathrm{C}^{1}((a, b)), p \in \mathrm{C}^{1}([0, \kappa])$ and $q \in \mathrm{C}^{1}([0, \kappa] \times(a, b))$, where either $l(a)=0$ or $l(b)=0$. Consider the differential equation

$$
\frac{d x}{d t}=p(t) l(x)+q(t, x) .
$$

(i) Assume that $q \equiv 0$. If either $\left.l\right|_{(a, b)} \neq 0$ or $\int_{0}^{\kappa} p(t) d t=0$, then the differential equation (16) has no limit cycles in region $[0, \kappa] \times(a, b)$.

(ii) Assume that $E$ is a non-empty set in $[0, \kappa],\left.q\right|_{E \times(a, b)} \neq 0$ and $\left.q\right|_{[0, \kappa] \times(a, b)} \geq 0(\leq$ $0)$. If $\operatorname{sgn}\left(\int_{0}^{1} p(s) d s\right) \operatorname{sgn}(l(x)) \operatorname{sgn}(q(t, x)) \geq 0$ when $(t, x) \in E \times(a, b)$, then the differential equation (16) has no periodic orbits in $[0,1] \times(a, b)$.

Proof. The lemma can be easily proved by comparing the solutions of the differential equation (16) with the solutions of the differential equation $d x / d t=p(t) l(x)$. For more details we refer the readers to the paper [27].

2.2. Limit cycles of a particular Abel differential equation. In this subsection we consider the Abel differential equation (4) with

$$
S(t, x)=\left(a_{1}(t) x-b_{1}(t)\right)\left(a_{2}(t) x-b_{2}(t)\right) x+\frac{1}{b_{1}(t)}\left(\dot{b_{1}}(t) x-\dot{a_{1}}(t) x^{2}\right),
$$

where $a_{i}, b_{i} \in \mathrm{C}^{\infty}([0, \kappa]), a_{i}(0)=a_{i}(\kappa), b_{i}(0)=b_{i}(\kappa)$ for $i=1,2$, and $b_{1}(t) \neq 0$.

One of the particularities of this Abel differential equation is that $a_{1}(t) x-b_{1}(t)=0$ is an invariant curve of it.

First we apply Lemma 5 and Theorem 6 to obtain some estimates for the number of limit cycles of the Abel differential equation (4) with $S(t, x)$ given in (17). In order to do that we consider the function

$$
F(t, x)=\alpha \ln |x|+\beta \ln \left|a_{1}(t) x-b_{1}(t)\right|-(\alpha+\beta+1) \ln \left|b_{1}(t)\right|+c(t),
$$

where $\alpha, \beta \in \mathbb{R}, c \in \mathrm{C}^{1}([0, \kappa])$ with $c(0)=c(\kappa)$. We shall denote $a_{1}(t), b_{1}(t), c(t)$ and $S(t, x)$ simply by $a_{1}, b_{1}, c$ and $S$. Then we have

$$
\begin{aligned}
& \frac{\partial F}{\partial t}(t, x)+S(t, x) \frac{\partial F}{\partial x}(t, x) \\
& \quad=\frac{\alpha S}{x}+\frac{\beta a_{1} S}{a_{1} x-b_{1}}+\frac{\beta\left(\dot{a_{1}} x-\dot{b_{1}}\right)}{a_{1} x-b_{1}}-(\alpha+\beta+1) \frac{\dot{b_{1}}}{b_{1}}+\dot{c}
\end{aligned}
$$




$$
\begin{gathered}
=\alpha\left(a_{1} x-b_{1}\right)\left(a_{2} x-b_{2}\right)+\beta a_{1}\left(a_{2} x-b_{2}\right) x-(\alpha+\beta) \frac{\dot{a_{1}}}{b_{1}} x-\frac{\dot{b_{1}}}{b_{1}}+\dot{c}, \\
\frac{\partial S}{\partial x}(t, x)=\left(a_{1} x-b_{1}\right)\left(a_{2} x-b_{2}\right)+a_{1}\left(a_{2} x-b_{2}\right) x+a_{2}\left(a_{1} x-b_{1}\right) x-2 \frac{\dot{a_{1}}}{b_{1}} x+\frac{\dot{b_{1}}}{b_{1}} .
\end{gathered}
$$

Thus a direct calculation shows that

$$
\begin{aligned}
\frac{\partial S}{\partial x}( & t, x)+\frac{\partial F}{\partial t}(t, x)+S(t, x) \frac{\partial F}{\partial x}(t, x) \\
= & (\alpha+\beta+3) a_{1} a_{2} x^{2}-\left((\alpha+\beta+2) a_{1} b_{2}+(\alpha+2) a_{2} b_{1}+(\alpha+\beta+2) \frac{\dot{a}_{1}}{b_{1}}\right) x \\
& +(\alpha+1) b_{1} b_{2}+\dot{c}
\end{aligned}
$$

Now we define

$$
V=\left\{\begin{array}{lr}
\left\{(t, x) \mid 0<x<b_{1} / a_{1}\right\} & \text { when } a_{1} b_{1}>0 \\
\left\{(t, x) \mid x>0 \text { or } x<b_{1} / a_{1}\right\} & \text { when } a_{1} b_{1}<0 \\
\left\{(t, x) \mid x>0, \text { and } x<b_{1} / a_{1} \text { for } t \text { with } a_{1} b_{1}>0\right\} & \text { when } a_{1} \text { has zeros }
\end{array}\right.
$$

and we have the next proposition.

Proposition 10. Suppose that $S$ is defined as in (17). Then the Abel differential equation (4) has at most 1 limit cycle (counted with multiplicity) in $V$, if one of the following conditions holds:

(i) $a_{1} \neq 0$ and there exists $\eta \in \mathbb{R}$ such that $a_{1} b_{2}+\eta a_{2} b_{1}+\dot{a_{1}} / b_{1} \geq 0(\leq 0)$.

(ii) $a_{2} \geq 0(\leq 0)$.

(iii) Either $a_{1} a_{2} \geq 0$ and $b_{1} b_{2} \leq 0$, or $a_{1} a_{2} \leq 0$ and $b_{1} b_{2} \geq 0$.

Proof. (i) Denote by $E_{1}=\left\{t \mid a_{1} b_{2}+\eta a_{2} b_{1}+a_{1} / b_{1} \neq 0\right\}$. We will divide the proof into three cases.

Case (i.a): $\eta=-1$. Firstly take transformation $y=a_{1} x / b_{1}$. Equation (4) is reduced to

$$
\dot{y}=\frac{b_{1}}{a_{1}} y(y-1)\left(a_{2} b_{1} y-a_{1} b_{2}-\frac{\dot{a_{1}}}{b_{1}}\right),
$$

and $V$ becomes $\widetilde{V}=\{(t, y) \mid 0<y<1\}$ (resp. $\widetilde{V}=\{(t, y) \mid y<0$ or $y>1\}$ ) when $a_{1} b_{1}>0$ $($ resp. $<0)$.

Observe that equation (21) can be rewritten as

$$
\dot{y}=\frac{a_{2} b_{1}^{2}}{a_{1}} y(y-1)^{2}-\frac{b_{1}}{a_{1}}\left(a_{1} b_{2}-a_{2} b_{1}+\frac{\dot{a_{1}}}{b_{1}}\right) y(y-1),
$$

where in each connected components of $\widetilde{V}$ we have

$$
\frac{b_{1}}{a_{1}}\left(a_{1} b_{2}-a_{2} b_{1}+\frac{\dot{a_{1}}}{b_{1}}\right) y(y-1) \geq 0(\leq 0) .
$$

When $E_{1}=\emptyset$ it follows from statement (i) of Lemma 9 that equation (21) has no limit cycles in $\widetilde{V}$, i.e. the Abel equation (4) has no limit cycles in $V$. 
When $E_{1} \neq \emptyset$ we claim that at most one connected component of $\widetilde{V}$ (resp. $V$ ) contains the limit cycles of (21) (resp. (4)). In fact this is trivial if $a_{1} b_{1}>0$. If $a_{1} b_{1}<0$, then

$$
\begin{aligned}
& \operatorname{sgn}\left(\int_{0}^{1} \frac{a_{2} b_{1}^{2}}{a_{1}} d t\right) \operatorname{sgn}\left(y(y-1)^{2}\right) \operatorname{sgn}\left(-\frac{b_{1}}{a_{1}}\left(a_{1} b_{2}-a_{2} b_{1}+\frac{a_{1}}{b_{1}}\right) y(y-1)\right) \\
& = \begin{cases}-\operatorname{sgn}\left(\int_{0}^{1} \frac{a_{2} b_{1}^{2}}{a_{1}} d t\right) \operatorname{sgn}\left(\frac{b_{1}}{a_{1}}\left(a_{1} b_{2}-a_{2} b_{1}+\frac{\dot{a}_{1}}{b_{1}}\right)\right) & y>1, t \in E_{1}, \\
\operatorname{sgn}\left(\int_{0}^{1} \frac{a_{2} b_{1}^{2}}{a_{1}} d t\right) \operatorname{sgn}\left(\frac{b_{1}}{a_{1}}\left(a_{1} b_{2}-a_{2} b_{1}+\frac{a_{1}}{b_{1}}\right)\right) & y<0, t \in E_{1} .\end{cases}
\end{aligned}
$$

Thus, by statement (ii) of Lemma 9 , either $\{(t, y) \mid y>1\}$ or $\{(t, y) \mid y<0\}$ contains no limit cycles of (21), which leads to our assertion.

On the other hand, take $\alpha=-1, \beta=-2$ and $c \equiv 0$ in (19). In each connected components of $V$ we get

$$
\frac{\partial S}{\partial x}(t, x)+\frac{\partial F}{\partial t}(t, x)+\frac{\partial F}{\partial x}(t, x) S(t, x)=\left(a_{1} b_{2}-a_{2} b_{1}+\frac{\dot{a_{1}}}{b_{1}}\right) x \geq 0(\leq 0) .
$$

Therefore when $E_{1} \neq \emptyset$ Lemma 5 implies that each connected component of $V$ has at most 1 limit cycle of the Abel equation (4) counted with multiplicity. Consequently, the number of limit cycles of (4) in $V$ is no more than 1.

Case (i.b): $\eta=0$. Similar to the argument in case (i.a) we change (4) to (21) and rewrite (21) into the form

$$
\dot{y}=\frac{a_{2} b_{1}^{2}}{a_{1}} y^{2}(y-1)-\frac{b_{1}}{a_{1}} y(y-1)\left(a_{1} b_{2}+\frac{\dot{a_{1}}}{b_{1}}\right) .
$$

It follows from Lemma 9 that there are no (resp. at most one) connected components of $V$ containing limit cycles of the Abel equation (4) when $E_{1}=\emptyset\left(\right.$ resp. $\left.E_{1} \neq \emptyset\right)$.

Now take $\alpha=-2, \beta=-1$ and $c=-\ln \left|a_{1}\right|$ in (19). In each connected components of $V$ we get

$$
\frac{\partial S}{\partial x}(t, x)+\frac{\partial F}{\partial t}(t, x)+\frac{\partial F}{\partial x}(t, x) S(t, x)=\left(a_{1} b_{2}+\frac{a_{1}}{b_{1}}\right)\left(x-\frac{b_{1}}{a_{1}}\right) \geq 0(\leq 0) .
$$

Thus from Lemma 5 and the above argument the Abel equation (4) has at most 1 limit cycle (counted with multiplicity) in $V$ when $E_{1} \neq \emptyset$.

Case (i.c): $\eta \neq-1,0$. Denote by

$$
\lambda_{1}=\max \left\{\frac{b_{1}}{a_{1}},-\eta \frac{b_{1}}{a_{1}}\right\}, \quad \lambda_{2}=\min \left\{\frac{b_{1}}{a_{1}},-\eta \frac{b_{1}}{a_{1}}\right\} .
$$

From assumption $\lambda_{1}>\lambda_{2}$ and $\lambda_{1}, \lambda_{2} \neq 0$. In addition we have that

$$
\begin{aligned}
& S\left(t, \frac{b_{1}}{a_{1}}\right)-\frac{d}{d t}\left(\frac{b_{1}}{a_{1}}\right)=0, \\
& S\left(t, \eta \frac{b_{1}}{a_{1}}\right)-\frac{d}{d t}\left(\eta \frac{b_{1}}{a_{1}}\right)=-\eta(\eta+1) \frac{b_{1}^{2}}{a_{1}^{2}}\left(a_{1} b_{2}+\eta a_{2} b_{1}+\frac{\dot{a_{1}}}{b_{1}}\right) \geq 0(\leq 0),
\end{aligned}
$$




$$
W\left(\frac{b_{1}}{a_{1}}, \eta \frac{b_{1}}{a_{1}}\right) \equiv 0
$$

where $W(\cdot, \cdot)$ represents the Wronskian determinant for two functions. So $4 \lambda_{1} \lambda_{2}\left(S\left(t, \lambda_{1}\right)-\right.$ $\left.\dot{\lambda_{1}}\right)\left(S\left(t, \lambda_{2}\right)-\dot{\lambda_{2}}\right)+W^{2}\left(\lambda_{1}, \lambda_{2}\right) \equiv 0$. According to Theorem 6 the Abel equation (4) has at most 2 non-zero limit cycles, counted with multiplicities. Note that $x=b_{1} / a_{1}$ is one of them and not located in $V$. Hence the conclusion holds.

(ii) Let $E_{2}=\left\{t \mid a_{2} \neq 0\right\}$. When $E_{2}=\emptyset$ we know that (4) is a Riccati equation and therefore it has at most 1 non-zero limit cycle. In what follows we consider the case that $E_{2} \neq \emptyset$.

Take $\alpha=\beta=-1$ and $c \equiv 0$ in (19). Then in each connected components of $V$ we have by assumption that

$$
\frac{\partial S}{\partial x}(t, x)+\frac{\partial F}{\partial t}(t, x)+\frac{\partial F}{\partial x}(t, x) S(t, x)=a_{2}\left(a_{1} x-b_{1}\right) x \geq 0(\leq 0) .
$$

In addition $\left.\left(a_{2}\left(a_{1} x-b_{1}\right) x\right)\right|_{V \cap\left(E_{2} \times \mathbb{R}\right)} \neq 0$. According to Lemma 5 the Abel equation (4) has at most 1 limit cycle in each connected components of $V$, counted with multiplicity.

When $a_{1} b_{1}>0$ or $a_{1}$ has zeros the conclusion clearly holds because $V$ is connected in these cases.

For the case $a_{1} b_{1}<0$ we again use (21) and rewrite the equation as

$$
\dot{y}=-\left(b_{1} b_{2}+\frac{\dot{a_{1}}}{a_{1}}\right) y(y-1)+\frac{a_{2} b_{1}^{2}}{a_{1}} y^{2}(y-1) .
$$

Following a way similar to the argument in case (i.a), we can verify using Lemma 9 that (21) has no limit cycles in $\{(t, y) \mid y>1\}$ or in $\{(t, y) \mid y<0\}$. That is all the limit cycles of the Abel equation (4) can only appear in one connected component of $V$, and therefore the number is at most 1 .

(iii) If $a_{1} a_{2} \equiv 0$ then (4) is reduced to a Riccati equation and therefore the number of non-zero limit cycle is no more than 1 . If $a_{1} a_{2} \not \equiv 0$ and $a_{1} \neq 0$, then $a_{2} \geq 0(\leq 0)$. The case goes back to statement (ii). The remaining case is $a_{1} a_{2} \not \equiv 0$ with $a_{1}$ having zeros. We know that $V$ is connected. Take $\alpha=-2, \beta=0$ and $c \equiv 0$ in (19). In $V$ we have by assumption that

$$
\frac{\partial S}{\partial x}(t, x)+\frac{\partial F}{\partial t}(t, x)+\frac{\partial F}{\partial x}(t, x) S(t, x)=a_{1} a_{2} x^{2}-b_{1} b_{2} \geq 0(\leq 0) .
$$

Moreover let $E_{3}=\left\{t \mid a_{1} a_{2} \neq 0\right\}$. Then $\left.\left(a_{1} a_{2} x^{2}-b_{1} b_{2}\right)\right|_{V \cap\left(E_{3} \times \mathbb{R}\right)} \neq 0$. Lemma 5 implies that the Abel equation (4) has at most 1 limit cycle $V$, counted with multiplicity.

Now we consider the non-existence of limit cycles for the Abel equation (4). Suppose that $S$ is defined as in (17). Rewrite the equation as

$$
\frac{d}{d t}\left(\frac{b_{1}}{x}\right)=-b_{1} \frac{\left(a_{1} x-b_{1}\right)\left(a_{2} x-b_{2}\right)}{x}+\dot{a_{1}} .
$$


Then for a non-zero periodic orbit $x=x(t)$ of the Abel equation (4) we have

$$
\int_{0}^{\kappa} b_{1}(t) \frac{\left(a_{1}(t) x(t)-b_{1}(t)\right)\left(a_{2}(t) x(t)-b_{2}(t)\right)}{x(t)} d t=0 .
$$

In particular note that $b_{1}\left(a_{1} x-b_{1}\right) / x \neq 0$ in $V$. Thus it is easy to obtain the following criterion.

Proposition 11. Suppose that $S$ is defined as in (17). Then the Abel equation (4) has no limit cycles in $V$ if $a_{2} x-b_{2}$ does not change sign in each connected component of $V$.

Proof. Denote by $\chi(t, x)=a_{2}(t) x-b_{2}(t)$. Suppose that $x=x(t)$ is a periodic solution of the Abel equation (4) in $V$. Then according to (23) and the assumption we have $\chi(t, x(t)) \equiv 0$. Note that the function $\chi$ is linear in the variable $x$ and the region $V$ is open. We get that $a_{2} \equiv 0$ and therefore $b_{2} \equiv 0$. Thus from (22) all the orbits of (4) in $V$ are periodic. Then the proposition is proved.

As we have explained in section 1 the limit cycles surrounding the origin of system (1) can be studied using the equation (6). Moreover these limit cycles in polar coordinates are all located in the region

$$
V_{1}=\left\{\begin{array}{lr}
{[0,2 \pi] \times \mathbb{R}^{+}} & \text {when } \psi>0, \\
\left([0,2 \pi] \times \mathbb{R}^{+}\right) \backslash\left\{(\theta, r) \mid 1+r^{n-1} \psi(\theta)=0\right\} & \text { when } \psi<0, \\
\left([0,2 \pi] \times \mathbb{R}^{+}\right) \backslash\left\{(\theta, r) \mid 1+r^{n-1} \psi(\theta) \leq 0\right\} & \text { when } \psi \text { has zeros. }
\end{array}\right.
$$

The transformation (5) sends the region $V_{1}$ into the region

$$
V_{2}=\left\{\begin{array}{lr}
\{(\theta, \rho) \mid 0<\rho<1 / \psi\} & \text { when } \psi>0, \\
\{(\theta, \rho) \mid \rho>0 \text { or } \rho<1 / \psi\} & \text { when } \psi<0, \\
\{(\theta, \rho) \mid \rho>0, \text { and } \rho<1 / \psi \text { for } \theta \text { with } \psi>0\} & \text { when } \psi \text { has zeros. }
\end{array}\right.
$$

Note that (6) is an Abel equation which can be rewritten as

$$
\frac{d \rho}{d \theta}=(\psi \rho-1)((n-1)(a \psi-\varphi) \rho-(n-1) a) \rho-\dot{\psi} \rho^{2}
$$

i.e. it is of the form (17) with

$$
a_{1}=\psi, \quad a_{2}=(n-1)(a \psi-\varphi), \quad b_{1}=1, \quad b_{2}=(n-1) a .
$$

Hence, as an application of the above propositions, we obtain the following result for system (1).

Proposition 12. Let $\varphi(\theta), \psi(\theta), \omega_{1}(\theta)$ and $\omega_{2}(\theta)$ be defined as in the abstract.

(i) Suppose there exist $\mu_{1}, \mu_{2} \in \mathbb{R}$ such that $\mu_{1}^{2}+\mu_{2}^{2} \neq 0$ and $\mu_{1} \omega_{1}+\mu_{2} \omega_{2} \geq 0(\leq 0)$. (i.a) If $\mu_{2}=0$, then system (1) has at most 1 limit cycle surrounding the origin.

(i.b) If $\mu_{2} \neq 0$ and $\psi \neq 0$, then system (1) has at most 1 limit cycle surrounding the origin.

Moreover when such a limit cycle exists, it is hyperbolic.

(ii) Suppose there exist $\nu_{1}, \nu_{2} \geq 0$ such that $\left(a \nu_{1}\right)^{2}+\nu_{2}^{2} \neq 0$ and $\omega_{1}\left(\nu_{1} a \psi-\nu_{2} \varphi\right) \leq 0$. (ii.a) If $\omega_{1} \varphi \equiv 0$, then system (1) has no limit cycles surrounding the origin. 
(ii.b) If $\omega_{1} \varphi \not \equiv 0$, then the following statements hold.

(ii.b.1) System (1) has no limit cycles surrounding the origin when $\psi>0$.

(ii.b.2) System (1) has at most 1 limit cycle surrounding the origin when $\psi<$ 0 . In addition, such a limit cycle exactly exists when $\omega_{1} \neq 0$.

(ii.b.3) System (1) has no limit cycles (resp. at most 1 limit cycle) surrounding the origin when $\psi$ has zeros and $\nu_{1} \leq \nu_{2}$ (resp. $\left.\nu_{1}>\nu_{2}\right)$.

Moreover when such a limit cycle exists, it is hyperbolic.

Proof. (i.a) By assumption we have $a \psi-\varphi=\omega_{1} \geq 0(\leq 0)$. According to (27) and statement (ii) of Proposition 10, equation (6) has at most 1 limit cycle in $V_{2}$, counted with multiplicity. That is system (1) has at most 1 limit cycle surrounding the origin, and this limit cycle is hyperbolic if it exists.

(i.b) Without loss of generality suppose that $\mu_{2}=1$. Take $\eta=\mu_{1} /(n-1)+1$. We get from (27) and by the assumptions that $a_{1}=\psi \neq 0$ and

$$
a_{1} b_{2}+\eta a_{2} b_{1}+\dot{a_{1}} / b_{1}=\eta(n-1)(a \psi-\varphi)+(n-1) a \psi+\dot{\psi}=\mu_{1} \omega_{1}+\omega_{2} \geq 0(\leq 0) .
$$

Therefore the number of limit cycles of $(6)$ in $V_{2}$ is at most 1 (counted with multiplicity) from statement (i) of Proposition 10. The conclusion immediately follows.

In order to prove statement (ii) first we observe that

$$
\begin{aligned}
\omega_{1}\left(\nu_{1} a \psi-\nu_{2} \varphi\right) & =\nu_{1}(a \psi-\varphi)^{2}+\left(\nu_{1}-\nu_{2}\right)(a \psi-\varphi) \varphi \\
& =\nu_{2}(a \psi-\varphi)^{2}+\left(\nu_{1}-\nu_{2}\right)(a \psi-\varphi) a \psi .
\end{aligned}
$$

Second we define the function

$$
\chi(\theta, \rho)=(a \psi-\varphi) \rho-a .
$$

From (27) $a_{2} \rho-b_{2}=(n-1) \chi$. In what follows we prove the statements one by one.

(ii.a) By assumption either $a \psi-\varphi=\omega_{1} \equiv 0$ or $\varphi \equiv 0$. Thus either $\chi \equiv-a$ or $\chi=a(\psi \rho-1)$. In any case we get that $\chi$ does not change sign in each connected component of $V_{2}$. Consequently equation (6) has no limit cycles in $V_{2}$ by Proposition 11. System (1) has no limit cycles surrounding the origin.

(ii.b) If $(a \psi-\varphi) \varphi=\omega_{1} \varphi \not \equiv 0$, then we have $\omega_{1} \not \equiv 0, \varphi \not \equiv 0$ and the following statements:

(i) $a \neq 0$ (otherwise $\nu_{2}>0$ and $\nu_{2} \varphi^{2} \leq 0$ by assumption, which implies $\varphi \equiv 0$ ).

(ii) $\nu_{1} \neq \nu_{2}$ (otherwise $\nu_{1}(a \psi-\varphi)^{2} \leq 0$ and therefore either $\nu_{1}=\nu_{2}=0$ or $\omega_{1} \equiv 0$ ).

Moreover due to the assumptions, (28) and (ii) we obtain

$$
\begin{array}{ll}
(a \psi-\varphi) \varphi \leq 0 \text { and }(a \psi-\varphi) a \psi \leq 0 & \text { when } \nu_{1}>\nu_{2}, \\
(a \psi-\varphi) \varphi \geq 0 \text { and }(a \psi-\varphi) a \psi \geq 0 & \text { when } \nu_{1}<\nu_{2} .
\end{array}
$$

(ii.b.1) In this case $V_{2}=\{(\theta, \rho) \mid 0<\rho<1 / \psi\}$. A direct calculation shows that $\chi(\theta, 0)=$ $-a$ and $\chi(\theta, 1 / \psi)=-\varphi / \psi$. Note that $a \neq 0$ from $(i)$ and $a \varphi / \psi \geq 0$ from (29). Since the function $\chi$ is linear in the variable $\rho,\left.\operatorname{sgn}(\chi)\right|_{V_{2}} \equiv-\operatorname{sgn}(a)$. By Proposition 11 equation (6) has no limit cycles in $V_{2}$, and therefore no limit cycles of system (1) surround the origin. 
(ii.b.2) When $\psi<0$ we have that $(i)$ leads to $a \psi \neq 0$. It is known by (29) that $\omega_{1}=a \psi-\varphi \geq 0(\leq 0)$. Hence statement (i.a) shows that system (1) has at most 1 limit cycle surrounding the origin, and such limit cycle is hyperbolic when it exists. Moreover, when $\omega_{1} \neq 0$ the equation $1+\psi r^{n-1}=a+\varphi r^{n-1}=0$ has no solutions and therefore the origin is the unique singularity of system (1). Recall that $\varphi \not \equiv 0$, and $a \varphi / \psi \geq 0$ from (29). According to Lemma 8 and a direct calculation the stabilities at the origin and at infinity of the system are the same. So such a limit cycle exactly exists.

(ii.b.3) If $\nu_{1}>\nu_{2}$, then (29) implies that either $(a \psi-\varphi) \psi \geq 0$ and $a<0$, or $(a \psi-\varphi) \psi \leq$ 0 and $a>0$. Together with (27) and statement (iii) of Proposition 10, equation (6) has at most 1 limit cycle in $V_{2}$, counted with multiplicity, i.e. system (1) has at most 1 limit cycle surrounding the origin, and this limit cycle is hyperbolic if it exists.

For the case that $\nu_{1}<\nu_{2}$ we again consider the sign of the function $\chi$ on $V_{2}$. Recall that $\chi$ is linear in the variable $\rho$ and $\chi(\theta, 0)=-a$. If we denote by $E^{+}=\{\theta \mid \psi>0\}$, $E^{-}=\{\theta \mid \psi<0\}$ and $E^{0}=\{\theta \mid \psi=0\}$, then

(iii) $\chi(\theta, 1 / \psi)=-\varphi / \psi$ when $\theta \in E^{+}$, and $a \varphi / \psi \geq 0$ from (29). Thus we have that $\left.\operatorname{sgn}(\chi)\right|_{\left(E^{+} \times \mathbb{R}^{+}\right) \cap V_{2}} \equiv-\operatorname{sgn}(a)$.

(iv) $\left.(-a)(a \psi-\varphi)\right|_{E^{-}} \geq 0$ by (29). Therefore $\left.\operatorname{sgn}(\chi)\right|_{\left(E^{-} \times \mathbb{R}^{+}\right) \cap V_{2}} \equiv-\operatorname{sgn}(a)$.

$\left.(v) \operatorname{sgn}(\chi)\right|_{\left(E^{0} \times \mathbb{R}^{+}\right) \cap V_{2}} \equiv-\operatorname{sgn}(a)$ by $(i v),(v)$ and the connectivity of $V_{2}$.

Consequently $\left.\operatorname{sgn}(\chi)\right|_{V_{2}} \equiv-\operatorname{sgn}(a)$. Applying Proposition 11 we get that equation (6) has no limit cycles in $V_{2}$, i.e. no limit cycles of system (1) surround the origin.

2.3. Application of the divergence formula. As shown in the proof of statement (i) of Proposition 10, only $a_{1} \neq 0$ can we use in the Abel equation to obtain the conclusion. For this reason we have to impose the restriction $\psi \neq 0$ in statement (i.b) of Proposition 12. Nevertheless with the divergence formula, given in Lemma 7, we are able to consider the case of $\psi$ having zeros in a simple and similar way.

First we note that the condition $\mu_{1}^{2}+\mu_{2}^{2} \neq 0$ and $\mu_{1} \omega_{1}+\mu_{2} \omega_{2} \geq 0(\leq 0)$ for system (1), actually leads to some "tangency" or "transversality" of the system on some curves. This observation is originated from the geometric condition of Theorem 6 and its application during the proof of statement (i) of Proposition 10. More precisely we have the following proposition.

Proposition 13. Let $\mathbf{v}$ be the vector field defined as in (2), and

$$
\gamma(\theta, r)=\left(\frac{\mu_{1}}{n-1}+2 \mu_{2}\right) \psi r^{n-1}+\left(\frac{\mu_{1}}{n-1}+\mu_{2}\right), \quad \text { with } \mu_{1}, \mu_{2} \in \mathbb{R}, \mu_{1}^{2}+\mu_{2}^{2} \neq 0 .
$$

Suppose that $\{(\theta, r) \mid \gamma(\theta, r)=0\} \neq \emptyset$. Then $\left.\left(D_{\mathbf{v}} \gamma\right)\right|_{\gamma=0}=r^{n-1}\left(\mu_{1} \omega_{1}+\mu_{2} \omega_{2}\right)$. Therefore if $\psi \not \equiv 0$ and $\mu_{1} \omega_{1}+\mu_{2} \omega_{2} \geq 0(\leq 0)$, then every periodic orbit of system (1) either does not intersect the curve $\gamma=0$, or it is a connected component of $\gamma=0$.

Proof. For convenience we denote by $\eta_{1}=\mu_{1} /(n-1)+2 \mu_{2}$ and $\eta_{2}=\mu_{1} /(n-1)+\mu_{2}$, i.e. $\gamma=\eta_{1} \psi r^{n-1}+\eta_{2}$. 
It is easy to check that $\eta_{1}=\eta_{2}=0$ if and only if $\mu_{1}=\mu_{2}=0$. Thus $\eta_{1}$ and $\eta_{2}$ are not simultaneously equal to zero. When $\eta_{1}=0$ and $\eta_{2} \neq 0$ the set $\{(\theta, r) \mid \gamma(\theta, r)=0\}$ is clearly empty. We only need to consider the case $\eta_{1} \neq 0$. A direct calculation shows that

$$
\begin{aligned}
\left.\left(D_{\mathbf{v}} \gamma\right)\right|_{\gamma=0} & =\left.\eta_{1}\left(\dot{\psi} r^{n-1}\left(1+\psi r^{n-1}\right)+(n-1) \psi r^{n-1}\left(a+\varphi r^{n-1}\right)\right)\right|_{\psi r^{n-1}=-\eta_{2} / \eta_{1}} \\
& =r^{n-1}\left(\dot{\psi}\left(\eta_{1}-\eta_{2}\right)+(n-1)\left(\eta_{1} a \psi-\eta_{2} \varphi\right)\right) \\
& =r^{n-1}\left(\mu_{1} \omega_{1}+\mu_{2} \omega_{2}\right) .
\end{aligned}
$$

So the first part of the proposition is proved.

In order to prove the second part we observe that $\gamma$ is a curve and $\left.\left(D_{\mathbf{v}} \gamma\right)\right|_{\gamma=0} \geq 0(\leq 0)$ from assumption. Thus an orbit of system (1) which intersects $\gamma=0$, either is contained in a connected component of $\gamma=0$, or crosses $\gamma=0$ and then stays in one connected component of $\gamma \neq 0$. Note that an orbit crossing $\gamma=0$ can not be periodic, so the conclusion follows.

Remark 14. Proposition 13 provides a geometrical meaning to conditions (I) and (III). More precisely the condition (I), i.e. the definite sign of $\mu_{1} \omega_{1}+\mu_{2} \omega_{2}$ with $\mu_{1}=1$ and $\mu_{2}=0$ implies that: The orbits of system (1) which intersect the curve $\psi r^{n-1}+1=0$, all go across the curve from the same side to the other side. This property was also mentioned by the authors of [16]. Nevertheless, for the condition (III) it seems that such similar property was never mentioned in the previous works. Actually condition (III) (the definite sign of $\omega_{2}$ ) means that: The orbits of system (1) which intersect the curve $2 \psi r^{n-1}+1=0$, all go across the curve from the same side to the other side. We would like to emphasize that, both of the two geometric properties are equivalent to the original conditions when $\psi<0$.

Now instead of the Abel equation (6) we shall use equation (3) for studying the limit cycles of system (1) when the function $\psi$ has zeros. Denote by $T$ the transformation of (5), i.e.

$$
T(\theta, r)=(\theta, \rho)=\left(\theta, \frac{r^{n-1}}{1+\psi(\theta) r^{n-1}}\right) .
$$

Let $V_{1}$ and $V_{2}$ be defined as in (24) and (25), respectively. Take $\mathbf{P}=(1, \mathcal{S}(\tau, \rho))$ and $\mathbf{Q}=(1, R(\theta, r))$, i.e. the vector fields associated to equations (6) and (3), respectively.

We begin to show the idea of finding a suitable auxiliary function for the differential equation (3).

Recall that Proposition 10, which is the main result for obtaining statement (i.b) of Proposition 12, is essentially based on Lemma 5, and for finding the auxiliary function " $F$ " (such as " $F "$ in (10) and (18)) for the Abel equation. In summary for equation (6) we shall use the auxiliary function

$$
F(\theta, \rho)=-\ln \left|(\psi \rho-1)\left(\kappa_{1} \psi \rho-\kappa_{2}\right) \rho\right|, \quad \text { with } \kappa_{1}, \kappa_{2} \in \mathbb{R}, \kappa_{1}^{2}+\kappa_{2}^{2} \neq 0 .
$$


So an essential reason in order that statement (i.b) of Proposition 12 holds is

$$
\left(\operatorname{div} \boldsymbol{P}+D_{\boldsymbol{P}} F\right)(\theta, \rho)=\frac{\partial \mathcal{S}}{\partial \rho}(\theta, \rho)+\frac{\partial F}{\partial \theta}(\theta, \rho)+\frac{\partial F}{\partial \rho}(\theta, \rho) \mathcal{S}(\theta, \rho) \geq 0(\leq 0) .
$$

Here $F$ is defined as in (30). On the other hand we can verify that $T$ is a diffeomorphism from $V_{1}$ to $V_{2}$. If we define

$$
\begin{aligned}
\bar{F} & =\ln |D T|+F \circ T \\
& =\ln \left|\psi r^{n-1}+1\right|-\ln \left|\eta_{1} \psi r^{n-1}+\eta_{2}\right|-\ln |r|-\ln (n-1),
\end{aligned}
$$

where $\eta_{1}=\kappa_{1}-\kappa_{2}$ and $\eta_{2}=-\kappa_{2}$, then (31) and Lemma 7 imply

$$
\frac{\partial R}{\partial r}(\theta, r)+\frac{\partial \bar{F}}{\partial \theta}(\theta, r)+\frac{\partial \bar{F}}{\partial r}(\theta, r) R(\theta, r)=\left(\operatorname{div} \boldsymbol{Q}+D_{\boldsymbol{Q}} \bar{F}\right)(\theta, r) \geq 0(\leq 0) .
$$

Thus when $\psi \neq 0$ the number of limit cycles of system (1) can also be estimated by applying Lemma 5 to equation (3) and using $\bar{F}$.

When the assumption $\psi \neq 0$ in statement (i.b) of Proposition 12 is changed by $\psi=0$ at some points, the inequality (31) is unknown because the functions $\lambda_{i}$ 's of Theorem 6 cannot be found. Nevertheless the divergence formula of Lemma 7 formally provides an auxiliary function $\bar{F}$ given in (32), which allows to verify the inequality (33) directly, and then to obtain the conclusion using Lemma 5. More precisely we have the next proposition.

Proposition 15. Suppose there exist $\mu_{1}, \mu_{2} \in \mathbb{R}$ such that $\mu_{1}^{2}+\mu_{2}^{2} \neq 0$ and $\mu_{1} \omega_{1}+\mu_{2} \omega_{2} \geq$ $0(\leq 0)$. If $\psi$ has zeros, then system (1) has at most 1 limit cycle surrounding the origin. Moreover, such a limit cycle is hyperbolic when it exists.

Proof. Due to the previous arguments we only need to consider the periodic orbits of the differential equation (3) which are located in the region $V_{1}$.

When $\psi \equiv 0$ equation (3) is reduced to a Bernoulli equation $d r / d \theta=a r+\varphi r^{n}$. Our claim can be directly checked from the expression of the general solution of this equation.

When $\psi \not \equiv 0$ take $\eta_{1}=\mu_{1} /(n-1)+2 \mu_{2}$ and $\eta_{2}=\mu_{1} /(n-1)+\mu_{2}$. Then by Proposition 13 each periodic orbit either does not intersect the curve $\eta_{1} \psi r^{n-1}+\eta_{2}=0$, or it is a connected component of this curve. Furthermore, since $\psi$ has zeros, the curve has no compact connected component. Hence such periodic orbits are all contained in one connected component of the region $U=V_{1} \backslash\left\{(\theta, r) \mid \eta_{1} \psi r^{n-1}+\eta_{2}=0\right\}$.

Now let $\bar{F}$ be the function determined by $(32)$. For $(\theta, r) \in U$ we get

$$
\begin{aligned}
\frac{\partial R}{\partial r}(\theta, r) & +\frac{\partial \bar{F}}{\partial \theta}(\theta, r)+\frac{\partial \bar{F}}{\partial r}(\theta, r) R(\theta, r) \\
& =-\frac{\mu_{1} \omega_{1}+\mu_{2} \omega_{2}}{\left(\psi r^{n-1}+1\right)\left(\eta_{1} \psi r^{n-1}+\eta_{2}\right)} r^{n-1} \geq 0(\leq 0) .
\end{aligned}
$$

Hence, if the set $E=\left\{\theta \mid \mu_{1} \omega_{1}+\mu_{2} \omega_{2} \neq 0\right\}$ is not empty, then by Lemma 5 the number of limit cycles of the differential equation (3) in $U$ is at most 1 . Moreover, when such a limit cycle exists, it is hyperbolic. 
For the case that $E=\emptyset$ we define a function

$$
\mathcal{F}(\theta, r)=\left(\eta_{1}-\eta_{2}\right) \ln \left|\eta_{1} \psi r^{n-1}+\eta_{2}\right|-(n-1) \eta_{1} \ln |r| .
$$

If $r=r(\theta)$ is an arbitrary orbit of (3) located in $U$, then a direct calculation shows that

$$
\begin{aligned}
\frac{d \mathcal{F}}{d \theta}(\theta, r(\theta)) & =\eta_{1}\left(\frac{(n-1)\left(\eta_{1} a \psi-\eta_{2} \varphi\right)+\left(\eta_{1}-\eta_{2}\right) \dot{\psi}}{\eta_{1} \psi r^{n-1}+\eta_{2}} r^{n-1}-(n-1) a\right) \\
& =\eta_{1}\left(\frac{\mu_{1} \omega_{1}+\mu_{2} \omega_{2}}{\eta_{1} \psi r^{n-1}+\eta_{2}} r^{n-1}-(n-1) a\right) \\
& \equiv-(n-1) a \eta_{1} .
\end{aligned}
$$

This means that $\mathcal{F}(0, r(2 \pi))-\mathcal{F}(0, r(0))=-2(n-1) a \eta_{1} \pi$. Consequently the differential equation (3) has no limit cycles in $U$. This completes the proof of the proposition.

2.4. The distribution of limit cycles of system (1). Until now we have studied the limit cycles surrounding the origin of system (1). Now we consider the existence of limit cycles which do not surround the origin.

Proposition 16. Any arbitrary periodic orbit of system (1) must surround the origin if one of the following conditions holds.

(i) There exist $\mu_{1}, \mu_{2} \in \mathbb{R}$ such that $\mu_{1}^{2}+\mu_{2}^{2} \neq 0$ and $\mu_{1} \omega_{1}+\mu_{2} \omega_{2} \geq 0(\leq 0)$.

(ii) There exist $\nu_{1}, \nu_{2} \geq 0$ such that $\left(a \nu_{1}\right)^{2}+\nu_{2}^{2} \neq 0$ and $\omega_{1}\left(\nu_{1} a \psi-\nu_{2} \varphi\right) \leq 0$.

Proof. First the periodic orbits of system (1) which do not surround the origin, are also simple closed curves with $r>0$ in the polar coordinates. Hence we can study such orbits using system (2) and its vector field $\mathbf{v}$. Moreover when $\psi \equiv 0$ we have $\mathbf{v}=\left(1, a r+\varphi r^{n}\right)$, which implies that each periodic orbit of the system must surround the origin. In what follows we put our attention to the case $\psi \not \equiv 0$.

(i) Let $\eta_{1}=\mu_{1} /(n-1)+2 \mu_{2}, \eta_{2}=\mu_{1} /(n-1)+\mu_{2}$ and

$$
g(\theta, r)=\frac{1}{\left(\eta_{1} \psi(\theta) r^{n-1}+\eta_{2}\right) r} .
$$

It follows from the assumptions that $\eta_{1}^{2}+\eta_{2}^{2} \neq 0$. Thus $g$ is a smooth function defined in the region $U=\left\{(\theta, r) \mid r>0, \eta_{1} \psi r^{n-1}+\eta_{2} \neq 0\right\}$. Furthermore in each connected component of $U$ we obtain

$$
\begin{aligned}
\operatorname{div}(g \mathbf{v}) & =\frac{\partial}{\partial \theta}\left(\frac{\psi r^{n-1}+1}{\left(\eta_{1} \psi r^{n-1}+\eta_{2}\right) r}\right)+\frac{\partial}{\partial r}\left(\frac{\varphi r^{n-1}+a}{\eta_{1} \psi r^{n-1}+\eta_{2}}\right) \\
& =-\frac{\mu_{1} \omega_{1}+\mu_{2} \omega_{2}}{\left(\eta_{1} \psi r^{n-1}+\eta_{2}\right)^{2}} r^{n-2} \geq 0(\leq 0) .
\end{aligned}
$$

Observe that $\eta_{1} \psi r^{n-1}+\eta_{2}=0$ is either a simple closed curve surrounding the origin, or a non-closed curve, or does not exist. By Proposition 13 each periodic orbit which does not surround the origin is located in a connected component of $U$. Hence if $\mu_{1} \omega_{1}+\mu_{2} \omega_{2} \not \equiv$ 0 (i.e. $\mu_{1} \omega_{1}+\mu_{2} \omega_{2}$ has at most finitely many zeros), then our conclusion holds by Bendixson-Dulac Theorem, see for instance Theorem 7.12 of [20]. If $\mu_{1} \omega_{1}+\mu_{2} \omega_{2} \equiv 0$, 
then $\operatorname{div}(g \mathbf{v}) \equiv 0$. There exists a first integral which only is not defined in the curve $\eta_{1} \psi r^{n-1}+\eta_{2}=0$, in other words it is defined in $U$. Thus the region $U$ contains no limit cycles which do not surround the origin of the system, and the proposition follows in this case.

(ii) When $\omega_{1} \equiv 0$ the conclusion follows from statement (i).

For the case that $\omega_{1} \not \equiv 0$ we can check that statements $(i),(i i)$ and (29) in the proof of Proposition 12 still hold by assumptions. Thus $\omega_{1} \psi \geq 0(\leq 0)$. Now denote by $\gamma=\psi r^{n-1}+1$. In each connected component of the curve $\gamma=0$, we have $\psi<0$ and therefore $\left.\omega_{1}\right|_{\gamma=0}$ does not change sign and has finitely many zeros. By Proposition 13 we get that

$$
\left.\left(D_{\mathbf{v}} \gamma\right)\right|_{\gamma=0}=(n-1) \omega_{1} r^{n-1} \geq 0(\leq 0) .
$$

Hence any periodic orbit of system (2) does not intersect the curve $\gamma=0$. Note that a singularity of the system is contained in $\gamma=0$ if it is not the origin. Consequently all periodic orbits of system (2) must surround the origin (this observation is also stated in [16]). Therefore statement (ii) holds.

\section{Proof of the main Results}

There are two goals in this section. The first is to provide the proofs of Theorems 1 and 3 , and of Corollaries 2 and 4 . The second is to give some examples for illustrating the application of our results.

Proof of Theorem 1. The distribution of the limit cycles of system (1) is obtained directly by statement (i) of Proposition 16. Together with statement (i) of Proposition 12 and Proposition 15, the system has at most 1 limit cycle, and such a limit cycle is hyperbolic when it exists.

Now we focus on the exact number of the limit cycles of system (1) when $\psi>0$.

First, by (2) the origin is the unique singularity of the system. Moreover, taking into account the sign of $a$ and Lemma 8 , the stabilities at the origin and at infinity of the system are opposite (resp. the same) if $a \int_{0}^{2 \pi}(\varphi / \psi) d \theta>0$ (resp. $\left.<0\right)$. In these cases it is easy to verify the exact number of the limit cycles of the system. Hence statement (a) and statement (b) hold except the case $a \int_{0}^{2 \pi}(\varphi / \psi) d \theta=0$.

Second, from the definition of the functions $\omega_{1}, \omega_{2}, \varphi$ and $\psi$ we have that

$$
\mu_{1} \omega_{1}+\mu_{2} \omega_{2}=\left(\mu_{1}+2(n-1) \mu_{2}\right) a \psi-\left(\mu_{1}+(n-1) \mu_{2}\right) \varphi+\mu_{2} \dot{\psi} .
$$

For the case that $a \int_{0}^{2 \pi}(\varphi / \psi) d \theta=0$, the proof is divided into two cases.

Case (i): $\mu_{1} \omega_{1}+\mu_{2} \omega_{2} \equiv 0$. We only discuss the case $\mu_{2} \neq 0$, the case $\mu_{1} \neq 0$ follows in a similar way. Thus $\omega_{2}=-\mu_{1} \omega_{1} / \mu_{2}$. Since the limit cycle must surround the origin, we consider equation (6) in the region $V_{2}$. Doing the transformation $y=\psi \rho$ the differential 
equation becomes

$$
\begin{aligned}
\dot{y} & =\frac{1}{\psi} y(y-1)\left((n-1) \omega_{1} y+(n-1) \omega_{1}-\omega_{2}\right) \\
& =\frac{\omega_{1}}{\psi} y(y-1)\left((n-1) y+\frac{\mu_{1}+(n-1) \mu_{2}}{\mu_{2}}\right),
\end{aligned}
$$

and $V_{2}$ goes over to $\widetilde{V}=\{(t, y) \mid 0<y<1\}$.

From (35) and $\mu_{1} \omega_{1}+\mu_{2} \omega_{2} \equiv 0$ we obtain that

$$
\left(\mu_{1}+2(n-1) \mu_{2}\right) 2 a \pi=\left(\mu_{1}+(n-1) \mu_{2}\right) \int_{0}^{2 \pi} \frac{\varphi(\theta)}{\psi(\theta)} d \theta,
$$

and by $(36)$ and $a \int_{0}^{2 \pi}(\varphi / \psi) d \theta=0$ we have the following three statements:

(i.a) If $\mu_{1}+2(n-1) \mu_{2} \neq 0$ and $\mu_{1}+(n-1) \mu_{2} \neq 0$, then $a=\int_{0}^{2 \pi}(\varphi / \psi) d \theta=0$ and therefore (36) becomes $\dot{y}=-(\varphi / \psi) y(y-1)\left((n-1) y+(n-1)+\mu_{1} / \mu_{2}\right)$.

(i.b) If $\mu_{1}+(n-1) \mu_{2}=0$, then (36) becomes $\dot{y}=(n-1)\left(\omega_{1} / \psi\right) y^{2}(y-1)$.

(i.c) If $\mu_{1}+2(n-1) \mu_{2}=0$, then (36) becomes $\dot{y}=(n-1)\left(\omega_{1} / \psi\right) y(y-1)^{2}$.

Note that from statement (i) of Lemma 9, the differential equation under the three previous statements has no limit cycles in $\widetilde{V}$. Hence system (1) has no limit cycles in this case.

Case (ii): $\mu_{1} \omega_{1}+\mu_{2} \omega_{2} \not \equiv 0$. We know by (35) that $n$ is odd (otherwise $\mu_{1} \omega_{1}+\mu_{2} \omega_{2}$ must change sign). So suppose without loss of generality that $n=2 k+1, k \in \mathbb{Z}$. In what follows we consider the following perturbation

$$
\begin{aligned}
& \frac{d x}{d t}=\left(a+\varepsilon_{1}\right) x-y+P_{n}(x, y)+\varepsilon_{2} x\left(x^{2}+y^{2}\right)^{k}, \\
& \frac{d y}{d t}=x+\left(a+\varepsilon_{1}\right) y+P_{n}(x, y)+\varepsilon_{2} y\left(x^{2}+y^{2}\right)^{k},
\end{aligned}
$$

of the differential system (1). Clearly system (37) is of the form (1). From the definition of the functions $\varphi, \psi, \omega_{1}$ and $\omega_{2}$, these functions for system (37) satisfy

$$
\begin{aligned}
& \varphi_{\varepsilon_{1}, \varepsilon_{2}}=\varphi+\varepsilon_{2}, \\
& \psi_{\varepsilon_{1}, \varepsilon_{2}}=\psi>0, \\
& \mu_{1} \omega_{1, \varepsilon_{1}, \varepsilon_{2}}+\mu_{2} \omega_{2, \varepsilon_{1}, \varepsilon_{2}}=\mu_{1} \omega_{1}+\mu_{2} \omega_{2}+\varepsilon_{1}\left(\mu_{1}+2(n-1) \mu_{2}\right) \psi+\varepsilon_{2}\left(\mu_{1}+(n-1) \mu_{2}\right) .
\end{aligned}
$$

We assume that system (1) has a hyperbolic limit cycle and we shall arrive to a contradiction. According to (35) and $a \int_{0}^{2 \pi}(\varphi / \psi) d \theta=0$, there are two subcases.

Subcase (ii.a): $\left(\mu_{1}+2(n-1) \mu_{2}\right) a \neq 0$ and $\left(\mu_{1}+(n-1) \mu_{2}\right) \int_{0}^{2 \pi}(\varphi / \psi) d \theta=0$. Observe that $\varepsilon_{1}\left(\mu_{1}+2(n-1) \mu_{2}\right) \psi\left(\mu_{1} \omega_{1}+\mu_{2} \omega_{2}\right) \geq 0$ for either $\varepsilon_{1}>0$ or $\varepsilon_{1}<0$. There exists a small $\varepsilon_{1} \neq 0$ such that $\left.(37)\right|_{\varepsilon_{1}, 0}$ has a hyperbolic limit cycle and

$$
\mu_{1} \omega_{1, \varepsilon_{1}, 0}+\mu_{2} \omega_{2, \varepsilon_{1}, 0}=\mu_{1} \omega_{1}+\mu_{2} \omega_{2}+\varepsilon_{1}\left(\mu_{1}+2(n-1) \mu_{2}\right) \psi \neq 0, \quad a+\varepsilon_{1} \neq 0 .
$$


Note that $\int_{0}^{2 \pi}(\varphi / \psi) d \theta=0$ in this case. We can choose $\varepsilon_{2} \neq 0$ sufficiently small such that $\left.(37)\right|_{\varepsilon_{1}, \varepsilon_{2}}$ has a hyperbolic limit cycle and

$$
\begin{aligned}
& \mu_{1} \omega_{1, \varepsilon_{1}, \varepsilon_{2}}+\mu_{2} \omega_{2, \varepsilon_{1}, \varepsilon_{2}} \neq 0, \\
& \left(a+\varepsilon_{1}\right) \int_{0}^{2 \pi} \frac{\varphi_{\varepsilon_{1}, \varepsilon_{2}}}{\psi_{\varepsilon_{1}, \varepsilon_{2}}} d \theta=\left(a+\varepsilon_{1}\right) \varepsilon_{2} \int_{0}^{2 \pi} \frac{1}{\psi} d \theta>0 .
\end{aligned}
$$

However as we have proved above (38) implies that the differential system (37) $\left.\right|_{\varepsilon_{1}, \varepsilon_{2}}$ has no limit cycles, this shows a contradiction. Therefore system (1) has no limit cycles.

Subcase (ii.b): $\left(\mu_{1}+2(n-1) \mu_{2}\right) a=0$ and $\left(\mu_{1}+(n-1) \mu_{2}\right) \int_{0}^{2 \pi}(\varphi / \psi) d \theta \neq 0$. It is a similar argument to subcase (ii.a). We have $\varepsilon_{2}\left(\mu_{1}+(n-1) \mu_{2}\right)\left(\mu_{1} \omega_{1}+\mu_{2} \omega_{2}\right) \geq 0$ for either $\varepsilon_{2}>0$ or $\varepsilon_{2}<0$. Thus choosing a suitable small $\varepsilon_{2} \neq 0$, (37) $\left.\right|_{0, \varepsilon_{2}}$ has a hyperbolic limit cycle and

$$
\begin{aligned}
& \mu_{1} \omega_{1,0, \varepsilon_{2}}+\mu_{2} \omega_{2,0, \varepsilon_{2}}=\mu_{1} \omega_{1}+\mu_{2} \omega_{2}+\varepsilon_{2}\left(\mu_{1}+(n-1) \mu_{2}\right) \neq 0, \\
& \int_{0}^{2 \pi} \frac{\varphi_{0, \varepsilon_{2}}}{\psi_{0, \varepsilon_{2}}} d \theta=\int_{0}^{2 \pi} \frac{\varphi+\varepsilon_{2}}{\psi} d \theta \neq 0 .
\end{aligned}
$$

Since $a=0$ in this case, there exists $\varepsilon_{1} \neq 0$ sufficiently small, such that $\left.(37)\right|_{\varepsilon_{1}, \varepsilon_{2}}$ has a hyperbolic limit cycle and

$$
\begin{aligned}
& \mu_{1} \omega_{1, \varepsilon_{1}, \varepsilon_{2}}+\mu_{2} \omega_{2, \varepsilon_{1}, \varepsilon_{2}} \neq 0 \\
& \left(a+\varepsilon_{1}\right) \int_{0}^{2 \pi} \frac{\varphi_{\varepsilon_{1}, \varepsilon_{2}}}{\psi_{\varepsilon_{1}, \varepsilon_{2}}} d \theta=\varepsilon_{1} \int_{0}^{2 \pi} \frac{\varphi+\varepsilon_{2}}{\psi} d \theta>0 .
\end{aligned}
$$

Again this still implies that the differential system (37) $\left.\right|_{\varepsilon_{1}, \varepsilon_{2}}$ has no limit cycles, which shows a contradiction. Consequently system (1) has no limit cycles. 0 .

In summary the differential system (1) has no limit cycles when $\psi>0$ and $a \int_{0}^{2 \pi}(\varphi / \psi) d \theta=$

Finally we provide three examples, the first one shows the existence and non-existence of limit cycles, and the second (resp. third) one shows the reachability of the upper bound of limit cycles when $\psi<0$ (resp. $\psi$ has zeros).

Consider system (1) with $a= \pm 1, n=2 k+1, P_{n}(x, y)=-(x+y)\left(x^{2}+y^{2}\right)^{k}$ and $Q_{n}(x, y)=(x-y)\left(x^{2}+y^{2}\right)^{k}$. Then a straightforward calculation shows that $\varphi=-1$, $\psi=1$ and

$$
\omega_{1}=2, \omega_{2}=6 k\left(\text { resp. } \omega_{1}=0, \omega_{2}=-2 k\right) \text { when } a=1(\text { resp. } a=-1) .
$$

Hence $\mu_{1} \omega_{1}+\mu_{2} \omega_{2}$ always does not change sign for arbitrary fixed $\mu_{1}, \mu_{2}$. In addition, $a \varphi / \psi=-1<0($ resp. $a \varphi / \psi=1>0)$ when $a=1$ (resp. $a=-1)$. From the theorem, the system has exactly 1 (resp. no) limit cycle when $a=1$ (resp. $a=-1$ ). Actually one can check that $x^{2}+y^{2}=1$ is a limit cycle of the system when $a=1$.

If we consider system (1) with $a=-1, n=2 k+1, P_{n}(x, y)=(x+2 y)\left(x^{2}+y^{2}\right)^{k}$ and $Q_{n}(x, y)=(-2 x+y)\left(x^{2}+y^{2}\right)^{k}$, then we get that

$$
\varphi=1, \quad \psi=-2, \quad \omega_{1}=1, \quad \omega_{2}=6 k .
$$


Similar to the previous example, the system has at most 1 limit cycle. Also one can check that $x^{2}+y^{2}=1$ is a limit cycle of the system. This verifies the reachability of the upper bound when $\psi<0$.

Take $a=-1, n=2 k+1, P_{n}(x, y)=x^{3}\left(x^{2}+y^{2}\right)^{k-1}$ and $Q_{n}(x, y)=y\left(2 x^{2}+y^{2}\right)\left(x^{2}+\right.$ $\left.y^{2}\right)^{k-1}$. Then

$$
\varphi=1, \quad \psi=\frac{1}{2} \sin (2 \theta), \quad \omega_{1}=-\frac{1}{2} \sin (2 \theta)-1, \quad \omega_{2}=-2 k \sin (2 \theta)+\cos (2 \theta)-2 k .
$$

Thus $-4 k \omega_{1}+\omega_{2}=2 k+\cos (2 \theta)>0$ and the number of limit cycle of the system is at most 1 . Observe that this system has a limit cycle $x^{2}+y^{2}=1$. The reachability of the upper bound is true when $\psi$ has zeros.

Proof of Theorem 3. This theorem follows directly from statement (ii) of Proposition 12 and statement (ii) of Proposition 16.

Now we show a concrete example with the existence and uniqueness of limit cycles. Consider system (1) with $a=-2, n=2 k+1, P_{n}(x, y)=(x+y)\left(x^{2}+y^{2}\right)^{k}$ and $Q_{n}(x, y)=(-x+y)\left(x^{2}+y^{2}\right)^{k}$. Then a straightforward calculation shows that

$$
\varphi=1, \quad \psi=-1<0, \quad \omega_{1}=1 \neq 0 .
$$

Hence $\omega_{1}\left(\nu_{1} a \psi-\nu_{2} \varphi\right) \leq 0$ holds taking $2 \nu_{1}-\nu_{2} \leq 0$. Using statement (iii) of the theorem, system (1) has exactly 1 limit cycle, which is hyperbolic and surrounds the origin. In fact one can check that $x^{2}+y^{2}=2^{1 /(2 k)}$ is a limit cycle of the system.

Using Theorems 1 and 3 we shall prove Corollaries 2 and 4 .

Proof of Corollary 2. Let $\mu_{1}=(n-1)(2 \mu-1)$ and $\mu_{2}=1-\mu$. Denote by $\gamma=\psi r^{n-1}+\mu$. From Proposition 13 we get that $\left(\mu_{1} \omega_{1}+\mu_{2} \omega_{2}\right) r^{n-1}=\left.\left(D_{\mathbf{v}} \gamma\right)\right|_{\gamma=0}$, where $\mathbf{v}$ is the vector defined as in (2). Since $\psi \neq 0$ and $\mu \neq 0$, the curve $\gamma=0$ is well-defined on the whole interval $[0,2 \pi]$. By assumption, either $\mu_{1} \omega_{1}+\mu_{2} \omega_{2} \equiv 0$ or $\mu_{1} \omega_{1}+\mu_{2} \omega_{2} \neq 0$. Observe that here $\mu_{1}^{2}+\mu_{2}^{2} \neq 0$. So by Theorem 1 the conclusion holds.

Proof of Corollary 4. The conclusion can be easily verified using Theorems 1 and 3, and a direct computation.

Here we provide three examples to illustrate the applications of our results. The first one compares our results with the previous results (I)-(IV) stated in the introduction section.

Example 1. Consider the differential system

$$
\begin{aligned}
& \frac{d x}{d t}=x-y-x^{3}+5 x^{2} y-x y^{2}-y^{3} \\
& \frac{d y}{d t}=x+y+3 x^{3}-x^{2} y+9 x y^{2}-y^{3} .
\end{aligned}
$$


Clearly it is of the form (1) with $a=1, n=3$ and

$$
\left(P_{3}, Q_{3}\right)=\left(-x^{3}+5 x^{2} y-x y^{2}-y^{3}, 3 x^{3}-x^{2} y+9 x y^{2}-y^{3}\right) .
$$

From direct computations we obtain that $\varphi=-1+4 \sin (2 \theta)$ and $\psi=2+\cos (2 \theta)$. Hence

$$
(n-1) a \psi+\dot{\psi}=4+2 \cos (2 \theta)-2 \sin (2 \theta)>0 .
$$

Applying statement (iii) of Corollary 4, the number of limit cycles of system (40) is at most 1. Moreover, as stated in the corollary, (41) means that $-2 \omega_{1}+\omega_{2}>0$. Therefore by Theorem 1 the system has exactly 1 limit cycle because

$$
a \int_{0}^{2 \pi} \frac{\varphi(\theta)}{\psi(\theta)} d \theta=\int_{0}^{2 \pi} \frac{-1+4 \sin (2 \theta)}{2+\cos (2 \theta)} d \theta=-\int_{0}^{2 \pi} \frac{1}{2+\cos (2 \theta)} d \theta<0 .
$$

Also for system (40) it follows from a direct calculation that

$$
\begin{aligned}
& \omega_{1}=3-4 \sin (2 \theta)+\cos (2 \theta), \\
& \omega_{1} \psi=(2+\cos (2 \theta))(3-4 \sin (2 \theta)+\cos (2 \theta)), \\
& \omega_{2}=10-10 \sin (2 \theta)+4 \cos (2 \theta) .
\end{aligned}
$$

Obviously all of these three equalities have indefinite signs, which violate the conditions of the results (I)-(IV). That is condition (iii) of Corollary 4 is indeed a new result.

In the second example we shall study the interval of the parameter $a$ in which the system has no limit cycles, or exactly one limit cycle.

Example 2. Consider the differential system

$$
\begin{aligned}
& \frac{d x}{d t}=a x-y+3 x^{3}-2 x^{2} y-x y^{2}-2 y^{3}, \\
& \frac{d y}{d t}=x+a y+2 x^{3}+5 x^{2} y+2 x y^{2}+y^{3} .
\end{aligned}
$$

It is of the form (1) with $n=3$ and

$$
\left(P_{3}, Q_{3}\right)=\left(3 x^{3}-2 x^{2} y-x y^{2}-2 y^{3}, 2 x^{3}+5 x^{2} y+2 x y^{2}+y^{3}\right) .
$$

One can check that $\varphi=2+\cos (2 \theta)$ and $\psi=2+\sin (2 \theta)$. Therefore we obtain

$$
(n-1) \varphi+\dot{\psi}=4+4 \cos (2 \theta) \geq 0 .
$$

Due to statement (iv) of Corollary 4 system (42) has at most 1 limit cycle counted with multiplicity. In addition, as stated in the corollary (43) is equivalent to $-4 \omega_{1}+\omega_{2} \geq 0$. Since

$$
a \int_{0}^{2 \pi} \frac{\varphi(\theta)}{\psi(\theta)} d \theta=a \int_{0}^{2 \pi} \frac{2+2 \cos (2 \theta)}{2+\sin (2 \theta)} d \theta=2 a \int_{0}^{2 \pi} \frac{1}{2+\sin (2 \theta)} d \theta
$$

from Theorem 1 it follows that the system has no (resp. exactly 1) limit cycle when $a \in[0,+\infty)($ resp. $a \in(-\infty, 0))$.

Finally we give the third example for showing the non-existence of limit cycles in a differential system. 
Example 3. Consider the differential system

$$
\begin{aligned}
& \frac{d x}{d t}=x-y+x y-2 y^{2}, \\
& \frac{d y}{d t}=x+y+2 x y+y^{2} .
\end{aligned}
$$

It is of the form (1) with $a=1, n=2$ and

$$
\left(P_{2}, Q_{2}\right)=\left(x y-2 y^{2}, 2 x y+y^{2}\right) .
$$

We have $\varphi=\sin \theta, \psi=2 \sin \theta$ and $\omega_{1}=\sin \theta$. If we take $\nu_{1}=1$ and $\nu_{2}=3$, then

$$
\omega_{1}\left(\nu_{1} a \varphi-\nu_{2} \varphi\right)=-\sin ^{2} \theta \leq 0
$$

By statement (iv) of Theorem 3 there is no limit cycle of system (44).

\section{ACKNOWLEDGEMENTS}

The first author is supported by the NSF of China (No.11401255) and the China Scholarship Council (No.201606785007) and the Fundamental Research Funds for the Central Universities (No.21614325). The second author is supported by the NSF of China (No.11771101) and the NSF of Guangdong Province (No.2015A030313669) and the Excellent Young Teachers Training Program for colleges and universities of Guangdong Province, China (No.Yq2013107). The third author is partially supported by a FEDER-MINECO grant MTM2016-77278-P, a MINECO grant MTM2013-40998-P, and an AGAUR grant number 2014SGR-568.

\section{REFERENCES}

[1] N.M.H. Alkoumi and P.J. Torres, Estimates on the number of limit cycles of a generalized Abel equation, Discrete Cont. Dyn.-A 31 (2011), 25-34.

[2] A. Álvarez, J.L. Bravo and M. Fernández, Limit cycles of Abel equations of the first kind, J. Math. Anal. Appl. 423 (2015), 734-745.

[3] M.J. Álvarez, J.L. Bravo and M. Fernández, Existence of non-trivial limit cycles in Abel equations with symmetries, Nonlinear Anal. Theor. Meth. Appl. 84 (2013), $18-28$.

[4] M.J. Álvarez, A. Gasull and H. Giacomini, A new uniqueness criterion for the number of periodic orbits of Abel equations, J. Differential Equations 234 (2007), 161-176.

[5] R. Benterki and J. Llibre, Limit cycles of polynomial differential equations with quintic homogeneous nonlinearities, J. Math. Anal. Appl. 407 (2013), 16-22.

[6] M. Blinov and Y. Yomdin, Generalized centre conditions and multiplicities for polynomial Abel equations of small degrees, Nonlinearity 12 (1999), 3869-3876.

[7] J.L. Bravo and M. Fernández, Limit cycles of non-autonomous scalar odes with two summands, Commun. Pure Appl. Anal. 12-2 (2013), 1091-1102.

[8] J.L. Bravo, M. Fernández and A. Gasull, Limit cycles for some Abel equations having coefficients without fixed signs, Int. J. Bifurcation and Chaos 19 (2009), 3869-3876.

[9] J.L. Bravo and J. Torregrosa, Abel-like differential equations with no periodic solutions, J. Math. Anal. Appl. 342 (2008), 931-942. 
[10] M. Briskin, J.P. Françoise and Y. Yomdin, The Bautin ideal of the Abel equation, Nonlinearity 11 (1998), 41-53.

[11] M. Carbonell and J. Llibre, Limit cycles of a class of polynomial systems, Proc. Royal Soc. Edinburgh 109A (1988), 187-199.

[12] L.A. Cherkas, Number of limit cycles of an autonomous second-order system, Differ. Uravn. 12 (1975), 944-946.

[13] A. Cima, A. Gasull and F. Mańosas, Limit cycles for vector fields with homogeneous components, Appl. Math. 24 (1997), 281-287.

[14] A. Cima and J. Llibre, Algebraic and topological classification of homogeneous cubic vector fields in the plane, J. Math. Anal. Appl. 147 (1990), 420-448.

[15] A. Cima, A. Gasull and F. Mañosas, Periodic orbits in complex Abel equations, J. Differential Equations 232 (2007), 314-328.

[16] T. Coll, A. Gasull and R. Prohens, Differential equations defined by the sum of two quasi-homogeneous vector fields, Can. J. Math. 49 (1997), 212-231.

[17] B. Coll and J. Llibre, Limit cycles for a quadratic system with an invariant straight line and some evolution of phase portraits, Colloquia Mathematica Societatis János Bolyai 53, Qualitative Theory of Differential Equations, Bolyai Institut, Szeged, Hungary (1988), 111-123.

[18] W.A. Coppel, Some quadratic systems with at most one limit cycle, Dynamics Reported 2 (1989), 61-88.

[19] J. Devlin, N.G. Lloyd and J.M. Pearson, Cubic systems and Abel equations, J. Differential Equations 147 (1998), 435-454.

[20] F. Dumortier, J. Llibre and J.C. Artés, Qualitative theory of planar differential systems, UniversiText, Springer-Verlag, New York, 2006.

[21] A. Gasull and A. Guillamon, Limit cycles for generalized Abel equations, Int. J. Bifurcation and Chaos 16 (2006), 3737-3745.

[22] A. Gasull, C. Li and J. Torregrosa, A new Chebyshev family with applications to Abel equations, J. Differential Equations 252 (2012), 1635-1641.

[23] A. Gasull and J. Llibre, Limit cycles for a class of Abel equation, SIAM J. Math. Anal. 21 (1990), 1235-1244.

[24] A. Gasull, J. Yu and X. Zhang, Vector fields with homogeneous nonlinearities and many limit cycles, J. Differential Equations 258 (2015), 3286-3303.

[25] J. Huang and H. Liang, An uniqueness criterion of limit cycles for the polynomial systems with homogeneous nonlinearities, J. Math. Anal. Appl., DOI:10.1016/j.jmaa.2017.08.008.

[26] J. Huang and H. Liang, Limit cycles of planar system defined by the sum of two quasi-homogeneous vecter fields, arXiv:1708.07959, preprint.

[27] J. Huang and H. Liang, A geometric criterion for equation $\dot{x}=\sum_{i=0}^{m} a_{i}(t) x^{i}$ having at most $m$ isolated periodic solutions, arXiv:1606.04776, preprint.

[28] J. Huang and Y. Zhao, Periodic solutions for equation $\dot{x}=A(t) x^{m}+B(t) x^{n}+C(t) x^{l}$ with $A(t)$ and $B(t)$ changing signs, J. Differential Equations 253 (2012), 73-99.

[29] Y. Ilyashenko, Hilbert-type numbers for Abel equations, growth and zeros of holomorphic functions, Nonlinearity $\mathbf{1 3}$ (2000), 1337-1342.

[30] A. Lins-Neto, On the number of solutions of the equation $d x / d t=\sum_{j=0}^{n} a_{j}(t) x^{j}$, $0 \leq t \leq 1$, for which $x(0)=x(1)$, Invent. Math. 59 (1980), 67-76. 
[31] R. Liouville, Sur une équation différentielle du premier ordre, (French) Acta Math. 27 (1903), 55-78.

[32] J. Llibre, Jesús S. Pérez del Río and J. A. Rodríguez, Structural stability of planar homogeneous polynomial vector fields: applications to critical points and to infinity, J. Differential Equations 125 (1996), 490-520.

[33] J. Llibre and G. Swirszcz, On the limit cycles of polynomial vector fields, Dyn. Contin. Discrete Impuls. Syst. 18 (2011), 203-214.

[34] J. Llibre and C. Valls, Classification of the centers, their cyclicity and isochronicity for a class of polynomial differential systems generalizing the linear systems with cubic homogeneous nonlinearities, J. Differential Equations 46 (2009), 2192-2204.

[35] J. Llibre and X. Zhang, Non-existence and uniqueness of limit cycles for quadratic polynomial differential systems, Proc. Roy. Soc. Edinburgh Sect. A, to appear.

[36] J. Llibre and X. Zhang, On the limit cycles of linear differential systems with homogeneous nonlinearities, Canad. Math. Bull. 58 (2015), 818-823.

[37] N.G. Lloyd, A note on the number of limit cycles in certain two-dimensional systems, J. London Math. Soc. 20 (1979), 277-286.

[38] N.G. Lloyd and J.M. Pearson, Bifurcation of limit cycles and integrability of planar dynamical systems in complex form, J. Phys. A: Math. Gen. 32 (1999), 1973-1984.

[39] A.A. Panov, The number of periodic solutions of polynomial differential equations, Math. Notes 64 (1998), 622-628.

[40] G.S. Rychkov, Limit cycles of the equation $u(x+1) d u=\left(-x+a x^{2}+b x u+c u+d u^{2}\right) d x$, Diff. Equations 8 (1972), 1748-1750.

${ }^{1}$ Department of Mathematics, Jinan University, Guangzhou 510632, P.R. China

E-mail address: thuangjf@jnu.edu.cn

2 School of Mathematics and Systems Science, Guangdong Polytechnic Normal University, Guangzhou 510665, P.R. China

E-mail address: lianghhgdin@126.com

3 Departament de Matemàtiques, Universitat Autònoma de Barcelona, 08193 Bellaterra, Barcelona, Catalonia, Spain

E-mail address: jllibre@mat.uab.cat 